\title{
On Doing Two Things at Once: III. Confirmation of Perfect Timesharing When Simultaneous Tasks Are Ideomotor Compatible
}

\author{
Anthony G. Greenwald \\ University of Washington
}

\begin{abstract}
A. G. Greenwald and H. G. Shulman (1973) found that 2 tasks characterized by ideomotor (IM) compatibility could be perfectly timeshared (i.e., performed simultaneously without mutual interference). The 2 tasks were pronouncing " $\mathrm{A}$ " or "B" in response to hearing those letter names, and making a manual left or right response to seeing a left- or right-positioned arrow. M.-C. Lien, R. W. Proctor, and P. A. Allen (2002) did not replicate Greenwald and Shulman's result, and concluded that their finding of perfect timesharing of 2 IM-compatible tasks might not be replicable. In the present research, Experiment 1 replicated Greenwald and Shulman's 1973 finding while also supporting the conclusion that Lien et al.'s nonreplication was due to their not instructing subjects to make 2 responses simultaneously in their timeshared task. Experiment 2 again replicated the perfect timesharing finding, using an alternative control procedure that mixed manual and vocal tasks in the same block.
\end{abstract}

This article reports the replication of a finding first reported 30 years ago. The replication was motivated by Lien, Proctor, and Allen's (2002; LP\&A hereafter) recent report of a nonreplication of Greenwald and Shulman's (1973; G\&S hereafter) finding of perfect timesharing of two simultaneous two-choice tasks when both tasks were ideomotor (IM) compatible. IM compatibility (Greenwald, 1972) is a relationship between stimuli and responses that was suggested by ideomotor theory (Greenwald, 1970c; James, 1890; Knuf, Aschersleben, \& Prinz, 2001).

Ideomotor theory proposes that responses are centrally coded by representations of their sensory feedback. Accordingly, it ought to be possible to select a response very directly, perhaps totally bypassing any limited-capacity process, by presenting a stimulus that closely resembles the response's sensory feedback. This should occur, for example, when a word is said in response to hearing it said. The dimension denoting the extent to which a stimulus corresponds to sensory feedback from its required response will be referred to as "ideomotor compatibility." (Greenwald, 1972, p. 52)

G\&S tested this hypothesis, that IM-compatible tasks could bypass a limited-capacity response selection process, by having subjects perform two IM-compatible choice tasks simultaneously (see also Greenwald, 1972). If IM-compatible tasks do indeed evade or minimize a response selection bottleneck (Pashler, 1990), then it may be possible for two IM-compatible tasks to be performed as rapidly when done simultaneously as when done separately. To test this hypothesized perfect timesharing, however, one cannot use just any pair of IM-compatible tasks. The tasks must be selected so that they avoid mutual interference by component processes other than response selection. For example, two IMcompatible tasks that both use visual stimuli might interfere with

This research was supported by National Institute of Mental Health Grants MH-41328, MH-01533, and MH-57672.

Correspondence concerning this article should be addressed to Anthony G. Greenwald, Department of Psychology, University of Washington, Box 351525, Seattle, Washington 98195-1525. E-mail: agg@u.washington.edu one another by requiring simultaneous attention to two locations. Similarly, two IM-compatible tasks that both use manual responses might produce mutual interference in response execution. G\&S avoided such extraneous sources of interference by using different stimulus modalities and different effector systems for the two tasks. One task used vocal responses to auditory stimuli (saying "A" or "B" in response to hearing these letter names). This task should have been IM-compatible because the letter-name stimuli closely resembled the auditory feedback from their required responses. The other task used manual responses to visual stimuli (moving a joystick switch to the left or right in response to a positioned arrow stimulus). This joystick task should have been IM-compatible because the stimuli incorporated the spatial aspect of kinesthetic or visual feedback from the required responses (see G\&S, p. 76).

G\&S's two experiments were psychological refractory period (PRP) experiments that included not only conditions in which the two tasks were simultaneous on each trial but also conditions in which the task stimuli were separated by interstimulus intervals (ISIs) ranging up to $1,000 \mathrm{~ms}$. In PRP experiments, slowest performances are expected when stimuli for the two tasks are simultaneous (ISI $=0$ ). A 1,000 -ms ISI condition (ISI $=1,000$ ) is often used as a control procedure in which the tasks are assumed to be separated sufficiently to avoid temporal overlap in their component information-processing stages.

\section{G\&S's Finding of Perfect Timesharing}

G\&S's findings confirmed the perfect timesharing prediction using, as dependent variable, the average latency for the two tasks. The reason for using average latency was that responses for the visual-manual task were generally faster at ISI $=0$ than at ISI $=$ 1,000 , whereas responses for the auditory-vocal task showed the reverse pattern. As a consequence, separate analyses of the two tasks would have led to mutually contradictory conclusions in regard to the perfect timesharing prediction.

In G\&S's Experiment 1, average latency for the two IMcompatible tasks with ISI $=0$ was $16 \mathrm{~ms}$ slower than the average 
latency with ISI $=1,000$. G\&S speculated that this small (and statistically nonsignificant) difference might have been due to some subjects adopting the strategy of responding to the visual stimulus before the auditory stimulus. Subjects might have used that strategy because the visual stimulus was nearly always first. Consequently, in G\&S's Experiment 2, subjects were instructed that the two stimuli would most often be simultaneous, and only ISIs of $0,100,200$, and $1,000 \mathrm{~ms}$ were used, in homogeneous-ISI blocks. With these revised conditions, average performance in the ISI $=0$ condition (average $=364 \mathrm{~ms}$ ) was $12 \mathrm{~ms}$ faster than in the ISI $=1,000$ condition (average $=376 \mathrm{~ms}$ ). That is, there was slightly better than perfect timesharing, although the difference was not statistically significant. G\&S concluded that, overall, their results were consistent with the hypothesis of perfect timesharing.

\section{Nonreplication by LP\&A}

Experiment 4 of LP\&A was designed to be a near-exact replication of G\&S's Experiment 2. However, LP\&A did not find perfect timesharing. Rather, they found that responses with ISI = 0 (average for two tasks $=476 \mathrm{~ms}$ ) were slower by an average of $34 \mathrm{~ms}$ than they were for ISI $=1,000$ (average $=442 \mathrm{~ms}$ ). ${ }^{1} \mathrm{In}$ LP\&A's Experiments 2 and 3, which differed from G\&S's Experiment 2 by using visual stimuli that lacked the spatial-position component of G\&S's stimuli, average performances for the ISI = 0 condition were, respectively, $50 \mathrm{~ms}$ and $60 \mathrm{~ms}$ slower than they were for ISI $=1,000$.

In summary, LP\&A's replications of G\&S's Experiment 2 found deviations from perfect timesharing that averaged $48 \mathrm{~ms}$ across their Experiments 2-4. This was in comparison to G\&S's report for their Experiment 2 of a 12-ms difference in the opposite direction (better performance for ISI $=0$ than for ISI $=1,000$ ). LP\&A pointed out that the G\&S result "has been cited widely as demonstrating that the PRP effect is eliminated when two tasks are ideomotor compatible because the responses are directly activated and bypass the normal response-selection processing" (p. 398). LP\&A interpreted their own findings as indicating that such characterizations of the G\&S result (e.g., De Jong, 1997; Meyer \& Kieras, 1997a; Pashler, 2000) should be regarded cautiously. LP\&A further indicated their doubt that G\&S's finding of perfect timesharing of IM-compatible tasks was replicable either "under the conditions in which [it was] obtained" (p. 405) or under "any conditions" (p. 408). LP\&A concluded that "the result of [G\&S's] Experiment 2 should be regarded as an anomaly unless subsequent research reveals conditions under which the absence of the PRP effect can be replicated" (p. 407). Clearly, LP\&A's results raised a serious question about the replicability of the main result of G\&S's Experiment 2 and, consequently, about the extent of the facilitatory role of IM compatibility in response selection. Their stated conclusions directly invited a replication of G\&S, which was the aim of the present research.

\section{Comparison of Procedures Between G\&S and LP\&A}

In an attempt to understand why LP\&A obtained results different from those of G\&S, the natural first strategy was to look for differences in procedures. Three procedural differences between the methods used by G\&S and those used by LP\&A might explain the difference in findings: (a) One of LP\&A's two IM-compatible tasks may not indeed have been IM compatible, (b) LP\&A's instructions to subjects did not stress the importance of responding both rapidly and simultaneously in the ISI $=0$ condition, and (c) unlike those of other studies that have obtained perfect timesharing, LP\&A's procedure did not use regularly spaced trials.

\section{IM Compatibility of Visual-Manual Task}

LP\&A described their manual response procedure as follows: "Participants were asked to grasp the joystick handle with their dominant hand and to place their other hand on the base of the joystick to stabilize it" (p. 398). The requirement to prevent the joystick base from moving would have obliged LP\&A's subjects to use the nondominant hand to exert a force opposed to that of the responding dominant hand. As a consequence, their joystick response required a two-hand coordination, and, importantly, the nondominant hand's role in this coordination opposed the IMcompatible direction-plus-position cue. By contrast, the joystick used by G\&S was fixed in position, requiring force to be exerted only by the dominant hand.

\section{Speed and Simultaneity Emphasis of Instructions}

In describing their instructions to subjects, LP\&A stated that they used "the instructions of [G\&S's] Experiment 2, which stated that most often the two stimuli would occur simultaneously" (p. 398). Their instructions, given to subjects preliminary to the experiment, were as follows: "Most often the two tasks will be presented simultaneously. Your job is to respond to each task as quickly and accurately as you can. Do not wait for the other task to appear. Remember that speed and accuracy are important" (M.-C. Lien, personal communication, July 3, 2002). Unfortunately, the instructions used by G\&S were not recorded in their article nor in any other documents that have survived the intervening 30 years. To the best of the present author's recollection, however, G\&S's instructions for Experiment 2 not only stressed the simultaneous occurrence of the stimuli but also encouraged subjects to respond both rapidly and simultaneously to the simultaneous stimuli. That recollection is consistent with G\&S's report that subjects had the incentive of a cash bonus for "fast and accurate performance" (p. 71). LP\&A's instructions, quoted above, mentioned simultaneity of tasks but did not urge simultaneity of responding. Conceivably, LP\&A's nonuse of instructions urging simultaneity of responding contributed to their nonreplication of G\&S.

An indication that LP\&A's subjects were not motivated to respond rapidly is provided by the reported latencies of performance for their IM-compatible condition. In the ISI $=0$ condition of LP\&A's Experiment 4 (their experiment that was most similar to G\&S's Experiment 2), IM-compatible latencies averaged 476

\footnotetext{
${ }^{1}$ LP\&A reported statistically significant rejection of the hypothesis of no differences in average latency among their four levels of ISI $(0,100$, 200 , and $1,000 \mathrm{~ms}$ ) but did not specifically report a test of the simple contrast of ISI $=0$ with ISI $=1,000$, which was not the largest difference between two ISIs. They described the 34-ms difference between ISI $=0$ and ISI $=1,000$ as "sizable" (LP\&A, p. 405), suggesting that the 34-ms contrast was itself statistically significant.
} 
ms, more than $100 \mathrm{~ms}$ slower than the 364-ms average in G\&S's Experiment 2.

\section{Fixed Versus Variable Trial Spacing}

In their experiments demonstrating perfect timesharing of IMcompatible tasks, G\&S used a fixed 4-s spacing of trials, as had Greenwald (1972). In a study that demonstrated perfect timesharing with only one of the two tasks being IM-compatible, Schumacher et al. (2001) used a trial spacing that deviated from being fixed only by the relatively small variations in subjects' individualtrial latencies (there was a fixed spacing between a subject's response and the next trial's stimulus). In contrast to these three studies in which the trial pace was very regular, LP\&A used a procedure in which trials were separated by intervals that varied unpredictably between about 2 and $4 \mathrm{s.}^{2}$ The irregular spacing of trials in LP\&A's procedure may have prevented their subjects from preparing optimally for the timeshared task combinations.

It was clearly worth determining whether LP\&A's nonreplication of the G\&S findings could be explained by one or more of the above-described differences in procedures. Because the possibility of perfect timesharing of two decisions is most powerfully and clearly tested in a simultaneous-task (ISI $=0$ ) condition, the present two replication experiments focused on reproducing the simultaneous-task condition of G\&S's Experiment 2. The present Experiment 1 examined the procedure that seemed most likely to be responsible for differences in findings, the stress on speed and simultaneity of responding used by G\&S but not by LP\&A. The critical comparison, as in both G\&S and LP\&A, was between an ISI $=0$ condition and an ISI $=1,000$ condition. Experiment 2 compared timeshared (ISI $=0$ ) performance with performance in a different comparison condition, a mixed-task condition in which the vocal and manual tasks occurred singly, in random sequence, within the same block.

\section{Experiment 1}

Experiment 1 was limited to IM-compatible tasks. In addition to the between-subjects variation of instructions, there was a withinsubjects variation of four tasks: visual-manual single task, auditory-vocal single task, dual tasks with ISI $=0$, and dual tasks with ISI $=1,000$.

\section{Method}

\section{Subjects}

Thirty-four undergraduate students at University of Washington (22 female, 12 male) participated in exchange for a small course credit for their undergraduate psychology course. All subjects were naive regarding hypotheses. Subjects were assigned alternately to the LP\&A instruction condition (Condition LPA; $n=17$ ) and to the G\&S instruction condition (Condition GS; $n=16$ ) on the basis of their order of arrival for the experiment. The reported data include all but 1 female participant who did not complete the experiment because her spoken responses were categorized with insufficient accuracy by the automatic speech-recognition software.

\section{Procedure}

Subjects participated individually in cubicles. The experimental session started with a test of the software's automatic scoring of the subject's vocal responses. Subjects were given a headset with earphones for auditory stimuli and a boom microphone to receive spoken responses. In the presence of an experimenter, subjects then saw instructions to pronounce the names of the letters $A$ and $B$, presented on a computer screen, each displayed eight times in random order. The software (Inquisit Version 1.32, 2002) automatically scored responses, displaying feedback of correct or error. If responses were not registered consistently by the speechrecognition software, the experimenter attempted to reposition the headset microphone, and the test sequence was repeated. Experimenters were asked to judge whether the speech-recognition software was working adequately and were instructed to terminate the experiment if it appeared that the software was not functioning adequately. One such termination was necessary.

Tasks. Stimuli for the visual-manual task were arrows positioned either left or right of screen center, pointing outward and downward at $45^{\circ}$ angles. On the 40-cm (diagonal) computer display, the centers of the two arrow positions were horizontally separated by $13.3 \mathrm{~cm}$. Each arrow was $2.8 \mathrm{~cm}$ long, with a tail width of $0.4 \mathrm{~cm}$. For the left arrow, the correct response was pressing a left-side key $(E)$ with left index finger, and for the right arrow, the correct response was pressing a right-side key $(I)$ with right index finger. ${ }^{3}$ Stimuli for the auditory-vocal task were the letter names "A" and " $\mathrm{B}$ ", pronounced in a male voice, presented from electronic files via the computer and earphones. Voice onset was within $10 \mathrm{~ms}$ of the file beginning, and the full duration of each file was $210 \mathrm{~ms}$. The letters were easily audible and easily distinguishable. Correct responses were to repeat the heard letter on each trial. Responses were scored for latency of onset and correctness by Inquisit Version 1.32 (2002)

Single-task conditions. The visual-manual and auditory-vocal tasks were administered in 16-trial blocks consisting of presentations of eight each of the two visual (arrow) stimuli or the two auditory (letter name) stimuli, respectively, in randomized order.

Dual-task conditions. In the dual-task ISI $=0$ condition, auditory and visual stimuli were presented simultaneously on each trial. Each block consisted of 64 trials, 16 each of the four possible combinations of auditory and visual stimuli, in random sequence. ${ }^{4}$ In the dual-task ISI $=1,000$ condition, each trial consisted of both a visual-manual and an auditoryvocal task, with onset of the auditory stimulus always 1,000 ms following the visual stimulus. There were 16 visual and 16 auditory stimuli in each

${ }^{2}$ The trial spacing for LP\&A's procedure was the variable sum of four durations: (a) the subject's response latency, (b) the experimenter's latency in giving a keyboard response to record the subject's vocal response or nonresponse on each trial, (c) 1,000 ms for error feedback if either the vocal or manual response was incorrect, and (d) an additional 1,000-ms pause. The interval from the start of one trial to the start of the next should therefore have varied between about 2 and $4 \mathrm{~s}$. Another feature of LP\&A's procedure that differed from G\&S's procedure was that LP\&A's experimenter remained in the company of the subject throughout the experiment in order to enter the subject's spoken responses on the computer keyboard.

${ }^{3}$ These keyboard responses preserved the left-right spatial variation of G\&S's joystick responses. The shared left-right spatial component of the arrow stimuli and the keyboard responses was the characteristic that justified regarding the task as IM compatible. The keyboard response was used to allow the present research to be readily replicated in any laboratory containing a desktop computer.

${ }^{4}$ The Inquisit Version 1.32 (2002) software was capable of registering and scoring only one response per trial in the dual-task ISI $=0$ condition. Accordingly, the software selected one response (manual or vocal) to record on each of these dual-task trials. This difference between trials was not discernible to the subject and therefore could not have influenced performance. With 64 trials in each dual-task block, the procedure allowed recording of latencies for 32 manual and 32 vocal responses per block. The experiment script is available on request from Anthony G. Greenwald. 
block. In both conditions, new trials started $2 \mathrm{~s}$ after the auditory stimulus of the preceding trial. This resulted in trials occurring at a fixed rate of every $2 \mathrm{~s}$ in the ISI $=0$ condition and every $3 \mathrm{~s}$ in the ISI $=1,000$ condition. ${ }^{5}$ To maintain these rates, the maximum latency allowed for vocal responses to auditory stimuli was $1,500 \mathrm{~ms}$.

Condition LPA. Based closely on the instructions used by LP\&A, the following instructions were presented in introductory instructions for the experiment: "During this experiment sometimes tasks will be presented simultaneously. Your job is to respond to each task as quickly and accurately as you can. Do not wait for the other task to appear. Remember that speed and accuracy are important." A reminder that "speed and accuracy are equally important" was provided before each block of trials for all single-task and dual-task conditions.

Condition GS. The instructions for Condition GS differed from those for Condition LPA in three ways: First, the preliminary instructions included "Throughout this experiment, it is important for you to respond as rapidly as you possibly can while maintaining a high rate of accuracy." Second, prior to each block of trials, subjects were reminded to respond "very rapidly." And, third, in the dual-task ISI $=0$ condition, the instructions prior to each block additionally reminded subjects "YOU ARE TO MAKE TWO RESPONSES AT THE SAME TIME.'

Overview of procedure. The experiment consisted of 16 blocks of trials, in four sets of 4 blocks. Each set of 4 blocks included 1 block of the visual-manual single-task condition, 1 block of the auditory-vocal singletask condition, 1 block of the ISI $=0$ dual-task condition, and 1 block of the ISI $=1,000$ dual-task condition. In each set of 4 blocks, the 2 single-task blocks preceded the 2 dual-task blocks. The order of the two single-task controls and that of the two dual tasks were randomized independently in each set of 4 blocks. Errors (vocal or manual) resulted in subjects seeing the word ERROR as feedback, in the lower center of the screen. The order of trials for each block was randomized independently for each subject and block.

Preliminary treatment of data. The full data set included a small percentage $(0.2 \%)$ of trials on which no response was registered within the maximum allowed period of $1,500 \mathrm{~ms}$. These trials were discarded. The vocal response received an unidentifiable code on $15.1 \%$ of vocal-response trials. Although these included some trials on which extraneous noises terminated the trial, it is a certainty that they were mostly trials on which a correct spoken response remained uncategorized by the speechrecognition software. The data were analyzed twice, once including these unrecognized responses and once excluding them. Results are reported for the analysis that retained the unidentified responses because of the likelihood that the great majority of them were correct responses. However, conclusions would be unaltered if the analysis that deleted these responses had been used instead.

Examination of response latencies revealed that $1.3 \%$ of all responses were faster than $100 \mathrm{~ms}$ and that $0.2 \%$ of all responses were slower than $1,000 \mathrm{~ms}$. Three analyses of correct response latencies were conducted, one using LP\&A's procedure of dropping trials that had latencies below $100 \mathrm{~ms}$ or above $2,000 \mathrm{~ms}$ (none in the present data set), one using narrower criteria for dropping trials (200 and 1,000 ms), and one using medians without dropping trials from the tails of the latency distributions. The reported analysis is the one that used LP\&A's procedure. However, data patterns were very similar for the other two analyses, and conclusions would not be altered if either of the other two analyses had been used instead.

\section{Results}

Figure 1 shows mean latencies of correct responses for Conditions LPA and GS, separately for manual responses, vocal responses, and their average. The data are also shown separately for the four successive sets of trial blocks that constituted internal replications of the design. Perhaps the most noticeable
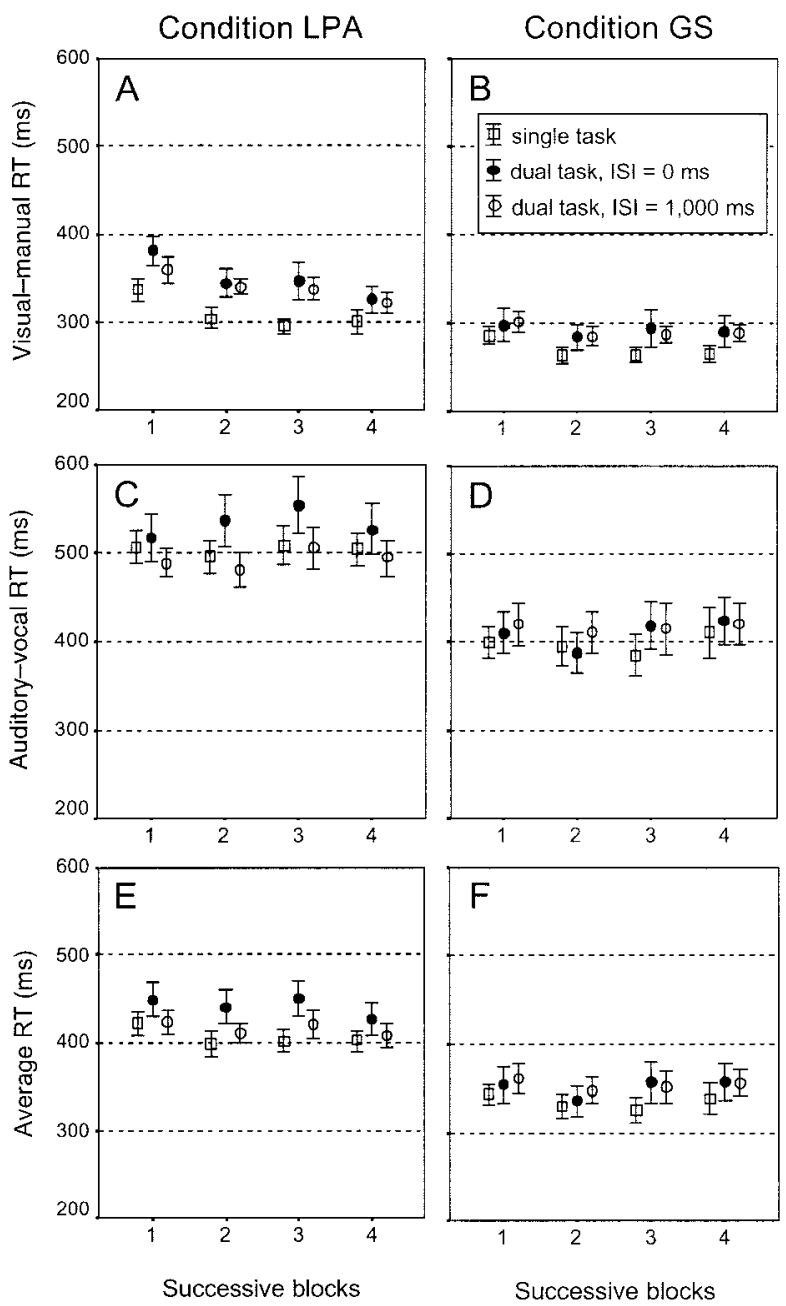

Figure 1. Mean latencies of correct responses for single-task and dualtask conditions of Experiment 1, shown for the visual-manual task (A and $\mathrm{B})$, the auditory-vocal task (C and D), and the average of both tasks (E and F). In Condition LPA $(n=17)$, instructions based on those used by Lien, Proctor, and Allen (2002) emphasized that stimuli for the interstimulus interval (ISI) $=0$ dual-task condition were simultaneous. In Condition GS $(n=16)$, instructions based on those used by Greenwald and Shulman (1973) urged rapid responding for all tasks and simultaneous responding in the ISI $=0$ condition. Error bars represent plus or minus one standard error of the mean. $\mathrm{RT}=$ response time.

result in Figure 1 is that performance was markedly faster in Condition GS than in Condition LPA. Vocal responses averaged $102 \mathrm{~ms}$ faster in Condition GS than in Condition LPA, and manual responses averaged $49 \mathrm{~ms}$ faster. This makes it clear that instructions had considerable impact on subjects' performance. The reduction in latency in Condition GS was accompanied by a relatively small increase in errors. Manual errors

5 These trial rates were faster than the fixed 4-s trial spacing used by G\&S. They also differed from the variable spacing of trials used by LP\&A (see Footnote 2). 
Table 1

Error Rate Percentages for Blocks 1-4 in Conditions LPA and GS of Experiment 1

\begin{tabular}{|c|c|c|c|c|c|c|c|c|}
\hline \multirow[b]{2}{*}{ Procedure and error type } & \multicolumn{4}{|c|}{ Condition LPA $(n=17)$} & \multicolumn{4}{|c|}{ Condition GS $(n=16)$} \\
\hline & 1 & 2 & 3 & 4 & 1 & 2 & 3 & 4 \\
\hline \multicolumn{9}{|l|}{ Single task } \\
\hline Manual errors & 0.74 & 6.62 & 1.84 & 0.00 & 1.17 & 3.52 & 3.57 & 3.13 \\
\hline Vocal errors & 8.51 & 7.50 & 8.46 & 10.10 & 7.42 & 9.77 & 9.38 & 9.38 \\
\hline Average errors & 4.62 & 7.06 & 5.15 & 5.05 & 4.30 & 6.64 & 6.47 & 6.25 \\
\hline \multicolumn{9}{|l|}{ Dual task, ISI $=0 \mathrm{~ms}$} \\
\hline Manual errors & 0.37 & 1.10 & 1.30 & 0.74 & 3.91 & 2.15 & 2.93 & 5.27 \\
\hline Vocal errors & 10.54 & 13.24 & 9.19 & 10.85 & 13.34 & 10.17 & 11.55 & 18.57 \\
\hline Average errors & 5.45 & 7.17 & 5.25 & 5.79 & 8.62 & 6.16 & 7.24 & 11.92 \\
\hline \multicolumn{9}{|l|}{ Dual task, ISI $=1,000 \mathrm{~ms}$} \\
\hline Manual errors & 4.04 & 0.39 & 0.37 & 0.37 & 0.39 & 1.95 & 1.95 & 0.39 \\
\hline Vocal errors & 12.66 & 11.76 & 10.02 & 6.25 & 11.56 & 8.98 & 12.50 & 8.20 \\
\hline Average errors & 8.35 & 6.08 & 5.20 & 3.31 & 5.98 & 5.47 & 7.23 & 4.30 \\
\hline
\end{tabular}

Note. Vocal error rates are inflated due to the speech-recognition software's miscategorization of some responses that were actually correct. Condition LPA = instruction condition from Lien, Proctor, and Allen (2002); Condition GS = instruction condition from Greenwald and Shulman (1973); ISI = interstimulus interval.

increased from an average of $1.5 \%$ to $2.5 \%$, and vocal errors increased from $9.9 \%$ to $10.9 \%{ }^{6}$

The greatest theoretical interest was in the comparison between the two dual-task conditions within each instructional condition. The data for ISI $=0$ and ISI $=1,000$ appear, respectively, in the second and third error bars in each group of three error bars in Figure 1. The hypothesis of perfect timesharing of two IMcompatible tasks would be supported by finding no difference between mean latencies in these two conditions.

Significance tests are presented here for overall condition means (as was done by LP\&A). In Condition GS, with instructions to respond rapidly at all times and simultaneously in ISI $=0$, latencies in the two ISI conditions were, on average, almost exactly the same (see Figures 1B, 1D, and 1F). For the visual-manual task, mean latencies were $291 \mathrm{~ms}$ for ISI $=0$ and $290 \mathrm{~ms}$ for ISI = $1,000, t(15)=-0.08, p=.94$. For the auditory-vocal task, mean latencies were $410 \mathrm{~ms}$ for ISI $=0$ and $416 \mathrm{~ms}$ for ISI $=1,000$, $t(15)=-0.71, p=.49$. For the average of the two tasks, the means were $351 \mathrm{~ms}$ for ISI $=0$ and $353 \mathrm{~ms}$ for ISI $=1,000$, $t(15)=-0.33, p=.75$.

The pattern was considerably different in Condition LPA. Here, with instructions stating that speed and accuracy were equally important and that stimuli would sometimes be simultaneous, latencies were generally slower for ISI $=0$ than for ISI $=1,000$. For the visual-manual task, mean latencies were $349 \mathrm{~ms}$ for ISI $=$ 0 and $339 \mathrm{~ms}$ for ISI $=1,000, t(16)=0.83, p=.42$. For the auditory-vocal task, mean latencies were $534 \mathrm{~ms}$ for ISI $=0$ and $491 \mathrm{~ms}$ for ISI $=1,000, t(16)=2.83, p=.01$. For the average of the two tasks, the means were $442 \mathrm{~ms}$ for ISI $=0$ and $415 \mathrm{~ms}$ for ISI $=1,000, t(16)=2.29, p=.04$.

The difference in pattern between Conditions GS and LPA for the two ISI conditions was itself statistically significant in the analysis of average latencies. For the interaction of this twocondition contrast with instructional condition, $F(1,31)=4.30$, $p=.04$. This result provides useful support for the conclusion that the instructional variation was important.

\section{Discussion}

Experiment 1 replicated the previous findings of both G\&S and LP\&A. G\&S reported that responding in their ISI $=0$ condition averaged $12 \mathrm{~ms}$ faster than in their ISI $=1,000$ condition; Condition GS of Experiment 1 yielded a very small difference (only 2 $\mathrm{ms}$ ) in the same direction. These differences were statistically nonsignificant in both G\&S and in Condition GS of the present experiment. LP\&A reported that responding in their ISI $=0$ condition was $34 \mathrm{~ms}$ slower than in their ISI $=1,000$ condition; the corresponding figure in Condition LPA of Experiment 1 was $27 \mathrm{~ms}$. These differences were statistically significant in both LP\&A and in Condition LPA of the present experiment.

One concern about interpreting findings from Experiment 1's Condition GS as showing perfect timesharing follows from there being higher error rates in Condition GS than in Condition LPA. If Condition GS's errors were greater for ISI $=0$ than for ISI $=$ 1,000 , then the perfect timesharing result could be a misleading appearance caused by speed-accuracy trade-offs. Indeed, error rates in Condition GS were higher for ISI $=0$ than for ISI $=$ 1,000 . The error rate for the visual-manual task was $3.6 \%$ for ISI $=0$ and $1.8 \%$ for ISI $=1,000, t(15)=3.05, p=.01$. For the auditory-vocal task, the error rate was $13.4 \%$ for ISI $=0$ and $10.3 \%$ for ISI $=1,000, t(15)=1.78, p=.10$. For the average error rate, the difference between the two conditions was statistically significant, $t(15)=2.67, p=.02$.

The error data are presented in detail in Table 1. Examination of these data revealed that error rates were especially high for the last (fourth) block of the dual-task procedure with ISI $=0$ in Condition GS. When the data were examined for just the first three blocks of the experiment, the difference in average error rates between ISI $=$ 0 and ISI $=1,000$ in Condition GS was considerably smaller and

\footnotetext{
${ }^{6}$ The error rate for vocal responding is certainly an overestimate. It includes an unknown number of correct responses that were incorrectly categorized by the speech-recognition software.
} 
nonsignificant, $t(15)=1.33, p=.20$. At the same time, the perfect timesharing result of Condition GS was maintained in the form of a nonsignificant 4-ms average superiority of ISI $=0$ over ISI $=$ $1,000, t(15)=-0.46, p=.65$. Also, the imperfect timesharing result of Condition LPA was maintained in the form of a significant 29-ms average superiority of ISI $=1,000$ over ISI $=0$, $t(16)=2.48, p=.03$.

In conclusion, Experiment 1 resolved the discrepancy in findings between $\mathrm{G} \& \mathrm{~S}$ and LP\&A. The differences in instructions can account for the difference in findings. Conceivably, had LP\&A used instructions that (a) elicited faster responding from their participants and (b) emphasized simultaneity of responding in ISI $=0$, they too would have found perfect timesharing of IMcompatible tasks.

In Condition GS, average performance in single-task blocks $(M=333 \mathrm{~ms})$ was faster than in the ISI $=0$ dual-task blocks $(M=$ $351 \mathrm{~ms}), t(15)=2.07, p=.06$. Given this observation, performance in Condition GS's ISI $=0$ dual-task blocks might be judged not to show perfect timesharing. That is, performance in dual-task blocks was inferior to performance of the same tasks in single-task blocks. This raises the question of what is the appropriate baseline to use in evaluating perfect timesharing. Experiment 2 provides new evidence bearing on that question.

\section{Experiment 2}

In prior research, ISI $=1,000$ dual-task procedures have been regarded as appropriate baseline conditions for use in PRP research because, in these procedures, the two tasks are separated sufficiently to remove concern about possible temporal overlap of information-processing stages for the two tasks. At the same time, the ISI $=1,000$ procedure involves task-switching (e.g., Jersild, 1927; Pashler, 2000; Rogers \& Monsell, 1995) on each trial. In the present research, as well as that of G\&S and LP\&A, the switching was between a visual-manual task and an auditory-vocal task. Task switching is known to incur costs that produce delays in responding. An ISI of 1,000 $\mathrm{ms}$ is not sufficiently long to eliminate task-switching costs (see Rogers \& Monsell, 1995, Figure 4).

Because it entails no task-switching cost, the single-task procedure might be seen as the appropriate baseline for comparison with simultaneous (i.e., ISI $=0$ ) dual-task performance. At the same time, the single-task procedure differs from the dual-task procedure in that it requires preparation for only two possible stimuli on each trial, in contrast to the dual-task procedure requiring preparation for four possible stimuli on each trial. This preparation difference has led researchers in the task-switching tradition to use task-repetition trials in mixed task blocks (i.e., blocks that include trials for both of the component tasks) rather than using pure single-task blocks to obtain baseline performance estimates for assessing task-switching costs.

Experiment 2 used both single-task and mixed-task comparison conditions to compare timesharing of two IM-compatible tasks with timesharing of two stimulus-response (SR)-compatible tasks. The instructions for these timesharing conditions encouraged speed and simultaneity of responding, as in Condition GS of Experiment 1 .

\section{Method}

\section{Subjects}

Twenty-seven undergraduate students at University of Washington (17 female, 10 male) participated in exchange for a small course credit for their undergraduate psychology course. All subjects were naive regarding hypotheses. Subjects were assigned alternately to Conditions SR (SRcompatible tasks; $n=14$ ) and IM (IM-compatible tasks; $n=13$ ) on the basis of their order of arrival for the experiment. The reported data include all participants.

\section{Procedure}

As in Experiment 1, the session started with a test of the software's automatic scoring of vocal responses. In the presence of an experimenter, subjects initially saw instructions to pronounce the names of a series of letters and numbers shown on the computer screen: $A, B$, one, and two, each displayed four times in random order. After establishing that the microphone was functioning adequately, the experimenter gave an oral instruction before leaving the subject alone to complete the experiment:

During parts of this experiment you will be asked to make two responses at once-one spoken and one on the computer keyboard. It is very important that you make both of these responses as rapidly as you can, while avoiding errors. You should try to make both responses at the same time, rather than making first one and then the other.

Condition IM. In Condition IM, stimuli for the visual-manual and auditory-vocal tasks were identical to those used in Experiment 1.

Condition SR. Responses for the visual-manual task in Condition SR were the same as for Condition IM. However, the stimulus for the left ( $E$ key) response was the word LEFT and the stimulus for the right ( $I$ key) response was the word RIGHT. These words were presented in the center of the screen, in Courier font, $0.7 \mathrm{~cm}$ in height. The two words were, respectively, approximately 1.2 and $1.5 \mathrm{~cm}$ in width. For the auditoryvocal task in Condition SR, the stimuli were the same as for Condition IM. However, the correct vocal response to heard "A" was "one," and the correct response to heard "B" was "two." Both of these tasks were considered to be SR-compatible, because each took advantage of very well-learned associations. Neither task, however, was IM-compatible, because neither task used stimuli that included components of feedback from their required responses.

Within each condition (IM and SR), subjects provided data for three types of tasks.

Single-task control. Each subject provided data for two single-task control procedures, one for the visual-manual task and the other for the auditory-vocal task. The single-task procedures differed from those used in Experiment 1 on the basis of previous findings that responses to a visual stimulus are faster when there is a simultaneous auditory stimulus than when the visual stimulus is presented alone (e.g., Bernstein, Clark, \& Edelstein, 1969). Using an irrelevant auditory stimulus simultaneously with visual stimuli in the single-task procedure therefore should assure that single-task performance is maximally fast. Accordingly, the visual-manual task included a click stimulus that was presented simultaneously with the arrows (Condition IM) or printed words (Condition SR). Correspondingly, an uninformative visual cue (three asterisks, centered in the display) was presented simultaneously with the auditory "A" and "B" stimuli in both auditory-vocal single tasks. Each single-task block had 32 trials.

Mixed-task control. A second control procedure mixed trials of the visual-manual and auditory-vocal tasks within blocks of trials. Whereas blocks of the single-task control procedures included 32 trials of one or the other of these tasks, mixed-task blocks included 64 trials, a random sequence of 32 trials each of the auditory-vocal and visual-manual tasks. 
As for the single-task procedure, visual-manual mixed-task trials included the uninformative simultaneous auditory (click) stimulus, and auditoryvocal trials included the visual asterisks.

Timeshared tasks. In the timeshared conditions, auditory and visual stimuli were presented simultaneously on each trial, identical to the dualtask ISI $=0$ procedure of Experiment 1 . Subjects were instructed to respond simultaneously and rapidly to the two stimuli on each trial, in the same fashion as for Condition GS of Experiment 1. Each block consisted of 64 trials, 16 each of the four possible combinations of auditory and visual stimuli.

Preliminary treatment of data. The collection of response latencies included a small percentage $(0.65 \%)$ of trials on which no response was registered within a maximum allowed period of $1,800 \mathrm{~ms}$, which was applied to maintain a fixed 2-s pace of trial presentations. These trials were discarded. The vocal response received an unidentifiable code on $10.2 \%$ of vocal-response trials. These included some trials on which extraneous noises terminated the trial, but they no doubt also included a substantial number of trials on which a correct vocal response went unrecognized by the speech-recognition software. As in Experiment 1, the reported analysis treated these unrecognized responses as correct responses. An additional analysis excluded these trials from latency analyses. Results from the two analyses did not differ in any consequential way.

As for Experiment 1, three analyses of correct response latencies were conducted, one using LP\&A's trial-deletion policy of dropping trials that had latencies outside the bounds of $100 \mathrm{~ms}(0.40 \%$ of trials in the present data) and 2,000 $\mathrm{ms}$ (not permitted by the present procedures), one using narrower criteria for dropping trials (200 and 1,000 ms), and one using medians without dropping trials from the tails of the latency distributions. Again, the reported analysis is the one that duplicated LP\&A's procedure. However, conclusions would not be altered if either of the other two analyses had been used.

\section{Results}

\section{Timesharing of IM-Compatible Tasks}

The right side of Figure 2 shows latencies for Condition IM. When the two tasks were performed simultaneously, responding was slightly slower than when the tasks were performed in the single-task control. For the visual-manual task, responding was faster for the single-task procedure $(M=273 \mathrm{~ms})$ than for the timeshared procedure ( $M=299 \mathrm{~ms}), t(12)=2.49, p=.03$. For the auditory-vocal task, single-task latencies $(M=349 \mathrm{~ms})$ were virtually identical to timeshared latencies ( $M=349 \mathrm{~ms}), t(12)=$ $0.01, p=.99$. For the average of both tasks, single-task responding (311 ms) was nonsignificantly faster than timeshared responding (324 ms), $t(12)=1.39, p=.19$. The faster responding for the visual-manual single task was accompanied by a higher error rate $(6.0 \%)$ than was found for the visual-manual timeshared task $(3.1 \%), t(12)=2.89, p=.01$.

Unexpectedly, responding was slower in the mixed-task control than in the timeshared task. This difference was statistically significant for the visual-manual and auditory-vocal tasks separately, as well as for the average of both (347 vs. $323 \mathrm{~ms}), t(12)=2.40$, $p=.03$.

Figures $2 \mathrm{~B}, 2 \mathrm{D}$, and $2 \mathrm{~F}$ provide the data for single, mixed, and timeshared tasks for successive quarters of the experiment (each task was performed in four separate blocks). With the exception of the first block of the visual-manual task, the approximate equality of single-task and timeshared performances was apparent from the beginning of the experiment. The inferiority of the mixed-task performance, relative to both timeshared and single-task perfor-
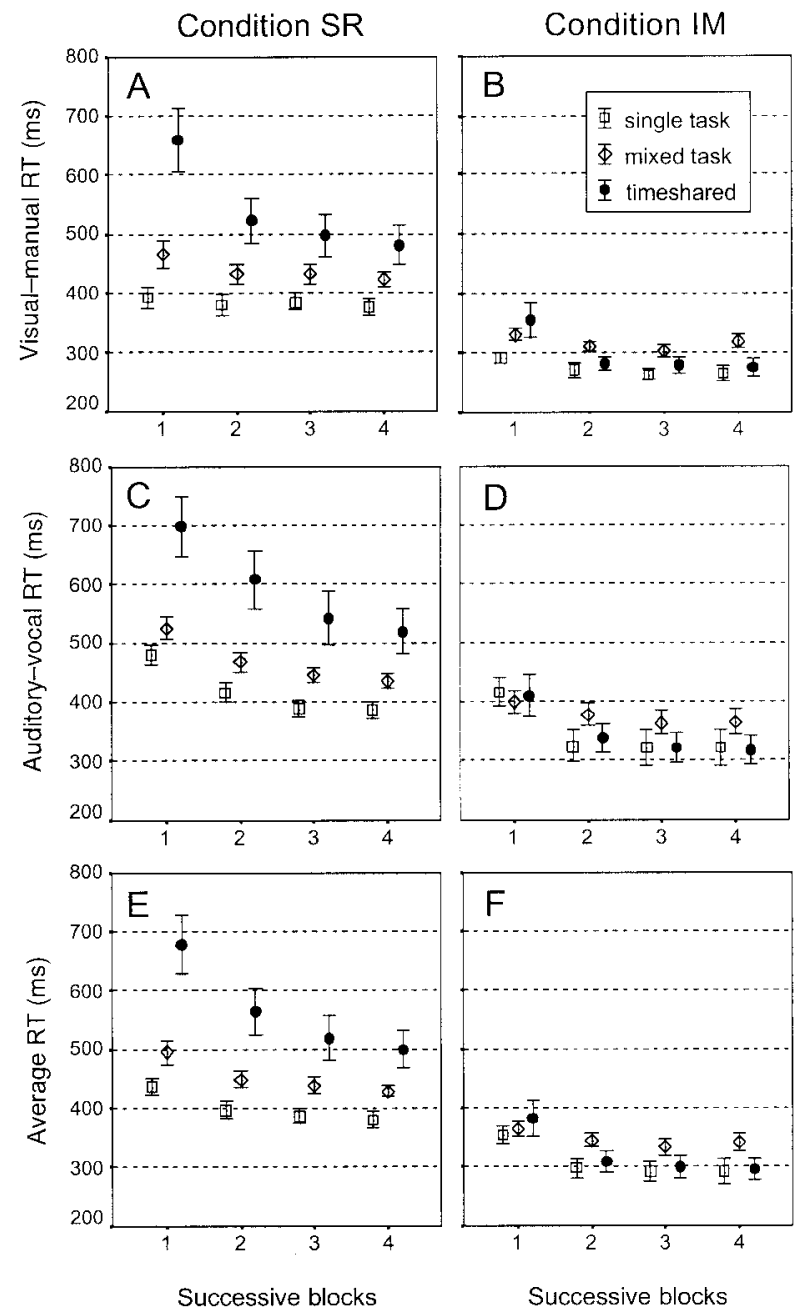

Figure 2. Mean latencies for single-task, mixed-task, and timeshared conditions of Experiment 2, shown for the visual-manual task (A and B), the auditory-vocal task (C and D), and the average of both tasks (E and F). Tasks were stimulus-response compatible in Condition SR $(n=14)$ and ideomotor compatible in Condition IM $(n=13)$. Error bars represent plus or minus one standard error of the mean. $\mathrm{RT}=$ response time.

mance, was generally apparent from the second through the fourth blocks.

\section{Timesharing of SR-Compatible Tasks}

Figures 2A, 2C, and 2E show latencies for Condition SR. These data show differences among the conditions that were (a) considerably larger than those observed in Condition IM and (b) qualitatively different in that slowest performance occurred for the timeshared task. For a comparison of the single-task control (mean average latency $=401 \mathrm{~ms}$ ) with the timeshared task (mean average latency $=562 \mathrm{~ms}$ ), the timesharing decrement averaged $161 \mathrm{~ms}$, $t(13)=5.98, p=.00005$. The timeshared SR-compatible task was also more than $100 \mathrm{~ms}$ slower than the mixed-task control $(M=$ $454 \mathrm{~ms}), t(13)=4.27, p=.001$. Figures $2 \mathrm{~A}, 2 \mathrm{C}$, and $2 \mathrm{E}$ reveal that these differences were consistently observed through all four blocks of the experiment. 


\section{General Discussion}

This research sought to determine the replicability of a result reported 30 years ago, perfect timesharing of two simultaneous IM-compatible tasks (Greenwald \& Shulman, 1973; G\&S). G\&S's result was recently called into question by a nonreplication (Lien, Proctor, \& Allen, 2002; LP\&A). Experiment 1 replicated G\&S's perfect timesharing finding when instructions for the ISI $=0$ dual task emphasized giving simultaneous and rapid responses to simultaneous stimuli. Also, it replicated LP\&A's finding of imperfect timesharing when instructions for the ISI $=0$ dual task indicated that stimuli would be simultaneous and stressed speed and accuracy equally. It can be concluded that both (a) the G\&S finding of perfect timesharing of IM-compatible tasks is replicable, and (b) replication of G\&S's finding depends on instructions to respond simultaneously in the dual-task condition with ISI $=0$.

Evidence critical for the conclusion of perfect timesharing was obtained from comparison of two dual-task conditions in Experiment 1 , one condition in which the stimuli for the two tasks were simultaneous (ISI $=0$ ) and a comparison condition in which onsets of the two stimuli were separated by $1 \mathrm{~s}($ ISI $=1,000)$. With IM-compatible visual-manual and auditory-vocal tasks and instructions to respond simultaneously (Condition GS), average response latencies were indistinguishable for the two dual-task conditions (actually slightly faster with ISI $=0$ than with ISI $=$ 1,000). When instructions did not stress responding simultaneously (Condition LPA), average latency of responses for the two tasks was significantly slower with ISI $=0$ than with ISI $=1,000$.

\section{Identifying the Appropriate Control Condition}

The conclusion that Experiment 1's results for ISI $=0$ indicate lack of mutual interference between two simultaneous IMcompatible tasks depends on accepting performance for ISI = 1,000 as an appropriate baseline. A problem with the baseline provided by the ISI $=1,000$ condition is that it requires task switching in the 1-s interval between the two stimuli. Such task switching is known to produce slowed responding on the second task.

An alternate baseline was available in the average performance of the two tasks in single-task blocks. Performance in the two single-task blocks averaged $18 \mathrm{~ms}$ faster than performance in the ISI $=0$ dual-task condition in Experiment 1 . However, it is a problem that, in the single-task conditions, subjects prepared for only two stimuli on each trial rather than four. To the extent that extra response preparation constitutes a burden that will increase latencies, single-task blocks do not provide an appropriate baseline.

Because of concern about the appropriateness of single-task performance as a baseline, Experiment 2 included an alternate baseline condition - a mixed-task procedure in which all four stimulus-response combinations of the visual-manual and auditory-vocal IM-compatible tasks appeared, randomly ordered with one stimulus per trial. This mixed-task procedure was assumed to match the response preparation burden of the timeshared IM-compatible condition, in which subjects had to be prepared for all four stimuli on each trial. It is interesting to note that performance in Experiment 2's mixed-task IM-compatible condition was significantly slower than in its timeshared condition-by an average of $24 \mathrm{~ms}$.
Experiment 2's mixed-task condition was decomposable into trials that repeated the (auditory-vocal or visual-manual) task of the previous trial and those that changed the task. As is known from the task-switching literature (e.g., Rogers \& Monsell, 1995), performance should be faster on repeat-task trials than on switchtask trials. In Experiment 2's IM-compatible mixed-task procedure, average latency on repeat-task trials was $333 \mathrm{~ms}$, and average latency on switch-task trials was $361 \mathrm{~ms}$. It is interesting to note that average performance that was faster than both of these values was observed in Experiment 2's timeshared condition (average = $322 \mathrm{~ms}$ ). Thus, if repeat-task trials of the mixed-task procedure are considered to provide an appropriate baseline for assessing timesharing efficiency for simultaneous tasks, the timesharing of IMcompatible tasks appears to be somewhat (nonsignificantly) better than perfect, $t(12)=1.28, p=.23$.

\section{Effects of Preparing to Inhibit?}

The average 11-ms inferiority of repeat-task trials in the mixedtask procedure relative to timeshared performance of the same tasks, even though not statistically significant, was surprising. Searching for an explanation for the slowness of mixed-task performance compared with timeshared performance led to consideration that, in performing the mixture of visual-manual and auditory-vocal tasks, subjects are obliged to inhibit either manual or vocal responding on each trial. That is, subjects are obliged not only to prepare to give either of the two vocal responses and either of the two manual responses but also to prepare to inhibit vocal or manual responding. Although this is a nonstandard interpretation, the mixed-task procedure can be understood as involving a preparation burden of six responses. In the IM-compatible condition, three of these six responses are vocal (saying "A," saying "B," or saying nothing), and three are manual (pressing $E$, pressing $I$, or pressing no key). By contrast, timeshared performance requires preparation of only four responses (saying “A," saying "B," pressing $E$, and pressing $I$ ), with no need to inhibit manual or vocal responding on any trial.

To obtain data on possible costs of preparing to inhibit responding, a small supplementary experiment compared performance of a two-choice IM-compatible task with performance of the same task interspersed randomly with $50 \%$ catch (no-response) trials. If the $50 \%$ catch-trial variation produced slower responding, it would be plausible to think that the mixed-task deficit observed in Experiment 2 reflected a cost of preparing to withhold responses.

To check this speculation, 10 additional subjects provided data for all four single tasks of Experiment 2, both the two IMcompatible tasks and the two SR-compatible tasks. Each of the four 2-choice tasks was conducted in one variation that presented a stimulus on each trial (100\% condition) and one that presented stimuli only on a random $50 \%$ of the trials (i.e., $50 \%$ catch trials). Performance was noticeably faster in the $100 \%$ condition. For SR-compatible tasks, mean latencies (averaged over vocal and manual tasks) were $504 \mathrm{~ms}$ and $455 \mathrm{~ms}$ for the $50 \%$ and $100 \%$ conditions, respectively, $t(9)=4.08, p=.003$. For IM-compatible tasks, the corresponding means were $423 \mathrm{~ms}$ and $370 \mathrm{~ms}, t(9)=$ $7.43, p=10^{-5}$. The approximately 50-ms slower average performance with $50 \%$ catch trials is consistent with the hypothesis that having to prepare to inhibit a response slows performance of that response. 


\section{Other Demonstrations of Perfect Timesharing}

Perfect timesharing with IM-compatible tasks has been reported not only in the 1973 G\&S study and the present research but also in an earlier study by Greenwald (1972). Perfect timesharing with other types of task combinations has been reported by Allport, Antonis, and Reynolds (1972) and by Schumacher et al. (2001). Allport et al. showed that adult subjects could simultaneously repeat continuous speech (IM compatible) and sight-read piano music (not IM compatible) without mutual interference. Schumacher et al. used two 3-choice tasks. One task was to press a left, middle, or right key in response to a similarly positioned visual stimulus (this was IM compatible), and the other task was to say "one," "two," or "three" in response, respectively, to low, medium, and high tones (this was not IM compatible). In Schumacher et al.'s study, perfect timesharing was not observed until the fifth session of the experiment, after approximately 1,600 trials of practice at each task. The perfect timesharing reported by Allport et al. and by Schumacher et al. may be understood as involving expert performance acquired through extensive practice. In contrast, the timesharing in the present research (and also in G\&S and in Greenwald, 1972) did not require practice-it was observed from the beginning of the experiment (see Figures 1B, 1D, and 1F and Figures $2 \mathrm{~B}, 2 \mathrm{D}$, and $2 \mathrm{~F}$ ).

Two recent theoretical analyses provide bases for expecting the possibility of perfect timesharing of non-IM-compatible tasks (Byrne \& Anderson, 2001; Meyer \& Kieras, 1997a, 1997b). Both of these analyses go beyond ideomotor theory in their ability to analyze many different timeshared task combinations. Both Meyer and Kieras's and Byrne and Anderson's analyses can readily accommodate the finding of perfect timesharing of two IMcompatible tasks. Therefore, the present results do not favor one of these formulations over the other. (Further, it is not obvious that these two theories should be regarded as competing with one another.)

\section{Temporal Locus of a Response-Selection Bottleneck}

The conception of a limited-capacity response-selection process was originally formulated in the context of the informationprocessing stage paradigm that was influential in cognitive psychology starting in the 1960s (see Smith, 1968). In most implementations of the stage paradigm, response selection is a limitedcapacity process that occurs after stimulus encoding and prior to response execution. Two of the present results-perfect timesharing of IM-compatible tasks and poorer performance of IMcompatible mixed tasks than single tasks-suggest a different conception of the temporal locus of response selection.

Greenwald's (1972) prediction of the possibility of perfect timesharing assumed that IM-compatible tasks might place no burden at all on a limited-capacity response-selection mechanism. However, if IM-compatible tasks entirely avoid a response-selection burden, then there should also be perfect timesharing when an IM-compatible task is timeshared with a non-IM-compatible task. Even though both Allport et al. (1972) and Schumacher et al. (2001) showed the possibility of perfect timesharing when only one of two tasks was IM-compatible, both G\&S and LP\&A showed that such perfect timesharing does not occur at low levels of practice. An alternative interpretation of the relation of IM compatibility to perfect timesharing can be offered by conceiving of response selection as done in large part by a preparation process that precedes stimulus presentation. This anticipatory preparation can be conceived as priming (or subthreshold activation) of the sensory and motor loci that are needed, respectively, to register the expected stimuli and to initiate the appropriate responses. This preparatory activation can be assumed to reduce the responseselection work that must be done after arrival of the stimulus. If the preparation includes a high level of activation of the task's needed sensorimotor pathways, then registration of a stimulus functions mainly as a trigger to activate the appropriate response. It may be especially easy to maintain high activation of sensorimotor pathways for IM-compatible tasks because of the (theorized) representational overlap between their sensory and motor sites.

An unexpected finding of the present research was the poorer performance in the mixed-task control procedure of Experiment 2 than in the same experiment's timeshared procedure for Condition IM. It is difficult to conceive of any explanation for this finding that limits the temporal span of response selection to the interval between stimulus presentation and response execution. Any such theory would face the seemingly impossible challenge of explaining why more response-selection work must be done to select one response than two. By contrast, the hypothesis that response selection involves activation prior to stimulus arrival easily explains this result. That is, because (for the mixed task) the subject must prepare to inhibit both vocal and manual responses, the sensorimotor paths for those responses may not be as strongly primed (i.e., maintained in as high a state of preparatory activation) as they are in the timeshared condition. This conception of response selection, as a mechanism that operates in part prior to stimulus presentation, is much in the spirit of the conception of attentional set, derived from the 19th-century concepts of Aufgabe (task attitude) and Einstellung (set; cf. Boring, 1950; Woodworth \& Schlosberg, 1954).

\section{Conclusion}

G\&S's demonstration of perfect timesharing was prompted by ideomotor theory's analysis of the role of representations of sensory feedback in response selection (Greenwald, 1970c). That theoretical analysis was supported by several experiments reported in the early 1970s (Greenwald, 1970a, 1970b, 1972; Greenwald \& Shulman, 1973). The validity of those older results was recently questioned by LP\&A but has now been bolstered by the present findings. Predictions of ideomotor theory have additionally been confirmed in a growing body of recent research (e.g., Bargh \& Chartrand, 1999; Hoffman, Sebald, \& Stoecker, 2001; Knuf et al., 2001; Kunde, 2001; Wegner, Ansfield, \& Pilloff, 1998).

It is a methodological cliché that nonreplications can have many causes. Examination of LP\&A's report suggested three possible causes-their use of instructions that did not stress the importance of responding rapidly or simultaneously on timeshared trials, their use of a visual-manual task that may not have been IM compatible, and their use of irregularly spaced trials that made stimulus presentations temporally unpredictable. The present experiments have established that LP\&A's instructions were likely responsible for not producing the (now reestablished as replicable) phenomenon of perfect timesharing of simultaneous IM-compatible tasks. 


\section{References}

Allport, D. A., Antonis, B., \& Reynolds, P. (1972). On the division of attention: A disproof of the single-channel hypothesis. Quarterly Journal of Experimental Psychology, 24, 225-235.

Bargh, J. A., \& Chartrand, T. L. (1999). The unbearable automaticity of being. American Psychologist, 54, 462-479.

Bernstein, I. H., Clark, M. H., \& Edelstein, B. A. (1969). Effects of an auditory signal on visual reaction time. Journal of Experimental Psychology, 80, 567-569.

Boring, E. G. (1950). A history of experimental psychology (2nd ed.). New York: Appleton-Century-Crofts.

Byrne, M. D., \& Anderson, J. R. (2001). Serial modules in parallel: The psychological refractory period and perfect time-sharing. Psychological Review, 108, 847-869.

De Jong, R. (1997). Compatibility effects on performance and executive control in dynamic task settings. In B. Hommel \& W. Prinz (Eds.), Theoretical issues in stimulus-response compatibility (pp. 223-239). Amsterdam: North-Holland.

Greenwald, A. G. (1970a). A choice reaction time test of ideomotor theory. Journal of Experimental Psychology, 86, 20-25.

Greenwald, A. G. (1970b). A double-stimulation test of ideomotor theory with implications for selective attention. Journal of Experimental Psychology, 84, 392-398.

Greenwald, A. G. (1970c). Sensory feedback mechanisms in performance control: With special reference to the ideomotor mechanism. Psychological Review, 77, 73-99.

Greenwald, A. G. (1972). On doing two things at once: Time sharing as a function of ideomotor compatibility. Journal of Experimental Psychology, 94, 52-57.

Greenwald, A. G., \& Shulman, H. G. (1973). On doing two things at once: II. Elimination of the psychological refractory period effect. Journal of Experimental Psychology, 101, 70-76.

Hoffmann, J., Sebald, A., \& Stoecker, C. (2001). Irrelevant response effects improve serial learning in serial reaction time tasks. Journal of Experimental Psychology: Learning, Memory, and Cognition, 27, 470482

Inquisit (Version 1.32) [Computer software]. (2002). Seattle, WA: Millisecond Software.

James, W. (1890). The principles of psychology (Vols. 1 \& 2). New York: Holt.
Jersild, A. T. (1927). Mental set and shift. Archives of Psychology, 14 (Whole No. 89), 5-82.

Knuf, L., Aschersleben, G., \& Prinz, W. (2001). An analysis of ideomotor action. Journal of Experimental Psychology: General, 130, 779-798.

Kunde, W. (2001). Response-effect compatibility in manual choice reaction tasks. Journal of Experimental Psychology: Human Perception and Performance, 27, 387-394.

Lien, M.-C., Proctor, R. W., \& Allen, P. A. (2002). Ideomotor compatibility in the psychological refractory period effect: 29 years of oversimplification. Journal of Experimental Psychology: Human Perception and Performance, 28, 396-409.

Meyer, D. E., \& Kieras, D. E. (1997a). A computational theory of executive cognitive processes and multiple-task performance: Part 1. Basic mechanisms. Psychological Review, 104, 3-65.

Meyer, D. E., \& Kieras, D. E. (1997b). A computational theory of executive cognitive processes and multiple-task performance: Part 2. Accounts of psychological refractory-period phenomena. Psychological Review, 104, 749-791

Pashler, H. (1990). Do response modality effects support multiprocessor models of divided attention? Journal of Experimental Psychology: $\mathrm{Hu}$ man Perception and Performance, 16, 826-842.

Pashler, H. (2000). Task switching and multitask performance. In S Monsell \& J. Driver (Eds.), Control of cognitive processes: Attention and performance XVIII (pp. 277-307). Cambridge, MA: MIT Press.

Rogers, R. D., \& Monsell, S. (1995). Costs of a predictable switch between simple cognitive tasks. Journal of Experimental Psychology: General, 124, 207-223.

Schumacher, E. H., Seymour, T. L., Glass, J. M., Fencsik, D. E., Lauber, E. J., Kieras, D. E., \& Meyer, D. E. (2001). Virtually perfect time sharing in dual-task performance: Uncorking the central cognitive bottleneck. Psychological Science, 121, 101-108.

Smith, E. E. (1968). Choice reaction time: An analysis of the major theoretical positions. Psychological Bulletin, 69, 77-110.

Wegner, D. M., Ansfield, M. E., \& Pilloff, D. (1998). The putt and the pendulum: Ironic effects of the mental control of action. Psychological Science, 9, 196-199.

Woodworth, R. S., \& Schlosberg, H. (1954). Experimental psychology (Rev. ed.). New York: Holt.

Received March 5, 2002

Revision received March 14, 2003

Accepted March 19, 2003

\section{Wanted: Your Old Issues!}

As APA continues its efforts to digitize journal issues for the PsycARTICLES database, we are finding that older issues are increasingly unavailable in our inventory. We are turning to our long-time subscribers for assistance. If you would like to donate any back issues toward this effort (preceding 1982), please get in touch with us at journals@apa.org and specify the journal titles, volumes, and issue numbers that you would like us to take off your hands. 